\title{
Evaluation of scattered-light spectra to apply the estimate of absorption spectra of flowers" petals
}

\author{
Hajime Imai ${ }^{\mathrm{a})}$, Mai Soeda, and Fumihiko Sekiguchi \\ Faculty of Science, Japan Women's University \\ 2-8-1 Mejirodai, Bukyo-ku, Tokyo 112-8681, Japan \\ a)imaih@fc.jwu.ac.jp
}

Abstract: We investigate the new method to evaluate the absorption spectrum by using the scattered light spectrum. The measurement set-up is to prevent the direct reflection light. By subtracting the two spectra, we can evaluate the change in the absorption spectra of samples where the transmitted light spectrum can not easily be measured. We apply this method to the derivation of absorption spectrum of flowers' petals, and estimate what kinds of absorption spectra contribute to the color of flowers' petals.

Keywords: spectroscopy, scattering spectrum, absorption spectrum Classification: Science and engineering for electronics

\section{References}

[1] M. D'Zmura and P. Lennie, "Mechanisms of color constancy," J. Opt. Soc. Am. A, vol. 3, no. 10, pp. 1162-1672, 1986.

[2] S. Tominaga and S. Ohashi, IPSJ J. (Japanese), vol. 33, no. 1, pp. 445450, 2005.

[3] M. Soeda, H. Imai, and F. Sekiguchi, "Recognition of petal colors in Petunia Hybrida from spectrum analysis," Optics Japan 2005, pp. 662663, 2005.

[4] G. Wyszecki and W. S. Stiles, "Color Science," Wiley, New York, 1982.

[5] Handbook of color science, University of Tokyo, Tokyo, 1998.

[6] R. J. Griesbach, S. Asen, and B. A. Leonnarat, "Petunia hybrida Antocyanins acylated with caffeic acid," Phytochemistry, vol. 30, no. 5, pp. 1729-1731, 1991.

[7] T. Ando, F. Tatsuzawa, N. Saito, M. Takahashi, Y. Tsunashima, H. Numajiri, H. Watanabe, H. Kokubun, R. Hara, H. Seki, and G. Hashimoto, "Differences in the floral anthocyanin content of red petunias and petunia exserta," Phytochemistry, vol. 54, pp. 495-501, 2000.

[8] K. Sekino, Master Thesis of Japan Women's University, (Japanese), 2004.

[9] K. Sekino and F. Sekiguchi, "HPLC analysis of pigments on the pedal of foundation species and F1 hybrid of Pechunia $\times$ hybrida," J. Japan Women's University Faculty of Science, vol. 14, pp. 53-58, 2006. 
[10] J. Nakajima, M. Yamazaki, and K. Saito, "Recent advances on reaction mechanism of anthocyanin biosythesis," Protein, Nuclear acid, and Enzyme, (Japanese), vol. 47, no. 3, pp. 217-224, 2002.

\section{Introduction}

The color of flowers' petals is determined by the absorption caused by the acylated form of pigments included in them. The absorption spectra are as yet obtained from the absorbance spectrum by using samples of dissolving mashed petals in solvent. However, it is difficult to evaluate the color of petals from those results.

Here we propose the new measurement method to estimate the absorption using the scattered light spectroscopy. It is well-known that the scattered light from the materials mainly contains the color information of them, while the direct reflected light contains the light-source information $[1,2]$. We have analyzed the scattered light spectrum to confirm it to correspond to the color of the materials, and have estimated the absorption spectrum. This process is nondestructive and it is possible to obtain the real absorption spectra of petals. The direct measurement of transmitted light spectra seems to be easy for the evaluation of the absorption. However, most samples of petals are too thick to measure the transmission light spectrum without changing the shape of samples. Our proposed method is applicable to any shape and any size of samples as they are.

\section{Measurement method and sample}

Figure 1 (a) shows the measurement set-up. To obtain the scattered light uniquely, we have optimized the angles of the irradiation and detection by changing each angle independently. Among the many combinations, we have selected the angles are $45^{\circ}$ and $0^{\circ}$ for irradiation and detection, respectively. This combination coincides with JIS Z 8701 and JIS Z 8722 of color evaluation. For the determination of this combination, we have measured the reflected light directivity as shown in Fig. 1(b) which is for the incident angle of $45^{\circ}$ [3]. The length of violet arrows corresponds to the detected light intensity. The detected light intensity for $45^{\circ}$ is the largest that means the detected light is due to the direct reflection. Other detected light intensities are for $0,15,30,60$, and $75^{\circ}$. The blue circle means the light intensity distribution for the random phase light: the scattered light. From this result, the detected lights for 0 and $15^{\circ}$ are considered to consist mainly of the scattered light. We have also checked that the obtained spectra have coincided with the color information of the samples [3], using the chromaticity diagram [4]. There is another method of using the polarizer to divide the scattered light and the reflected light. Here we will simultaneously evaluate the color of samples. Therefore, we select this method after JIS standards. The light sources are the tungsten lamp for visible wavelength region and the Xenon 

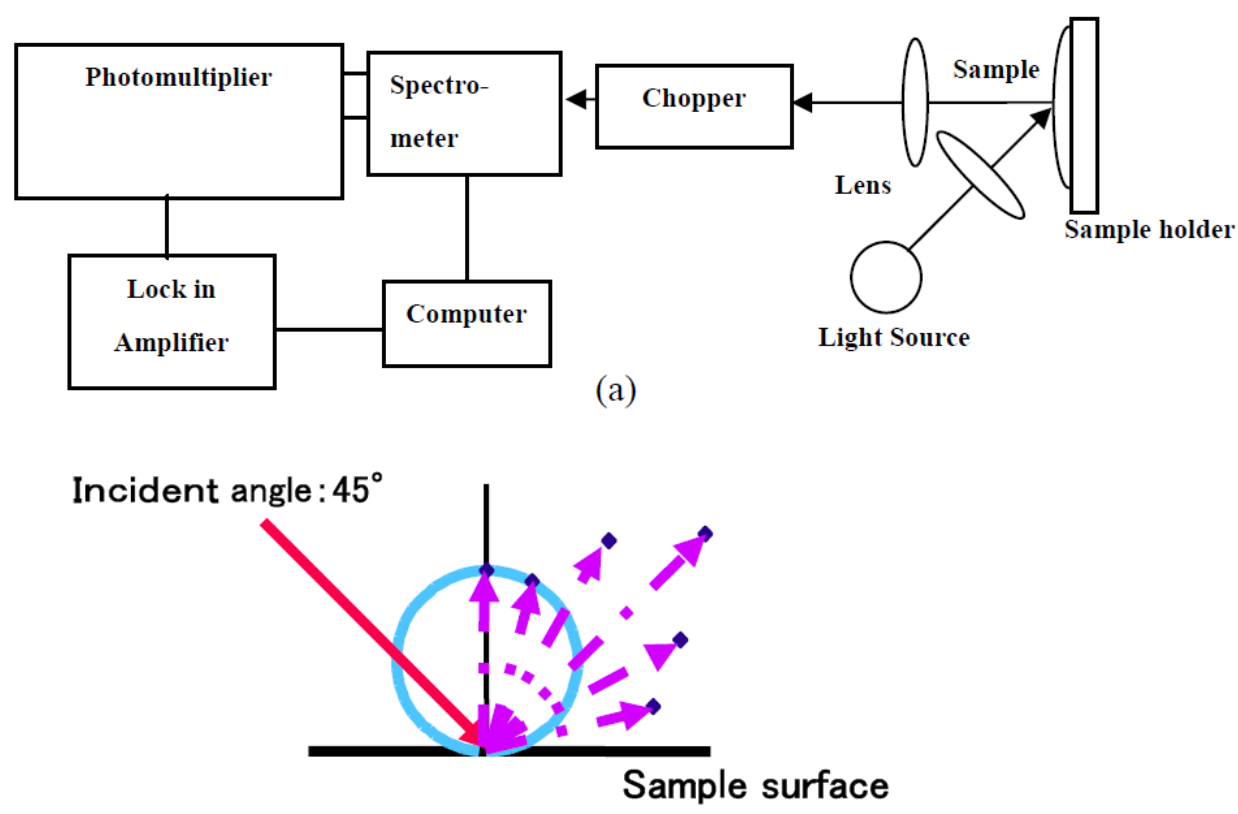

(b)

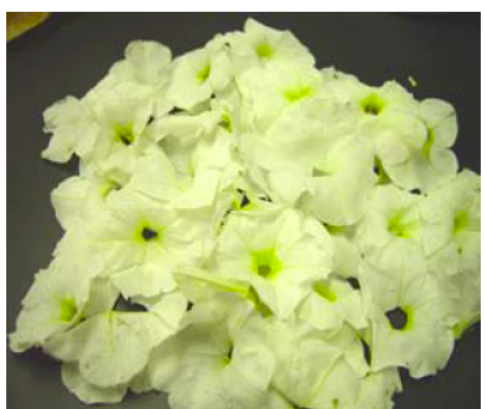

(c)

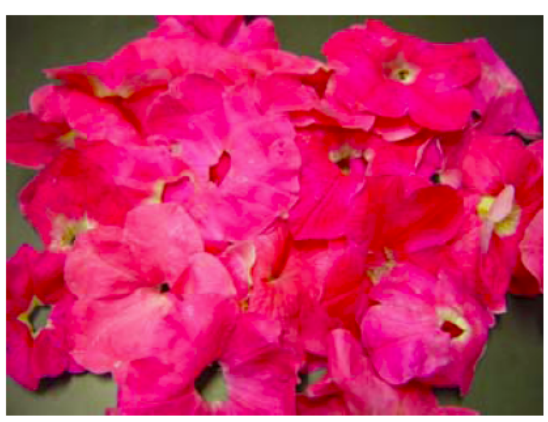

(d)

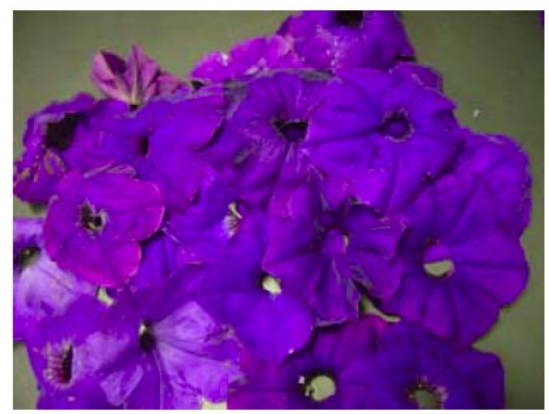

(e)

Fig. 1. (a) Measurement set-up, (b) the directivity of detected light intensity for the incident light angle $45^{\circ}$, and photographs of (c) light yellow, (d) red, and (e)violet petals of petunia hybrida

lamp near ultraviolet wavelength region.

Samples are the petals of petunia hybrida whose colors are light yellow, red, and violet. The photographs of the petals are shown in Fig. 1 (c)-(e). We picked up the single petal for each color and made it flat to place against the 
light source. The scattered light is converged through convex quartz lenses into the spectrometer, as shown in Fig. 1 (a).

\section{Measured results and discussions}

The measured scattered spectra are shown in Fig. 2 (a). These spectra are normalized by the irradiated light spectrum. The spectrum for the light yellow petal is almost flat in the visible wavelength region, the spectrum for the red petal has the large dip around the $550 \mathrm{~nm}$ wavelength, and that for violet has another dip around $660 \mathrm{~nm}$ wavelength. These spectra do not change against a slight change in the detection angle. This means these petals do not show the characteristics such as liquid crystals. These results suggest the absorption levels exist around these wavelengths of dips.

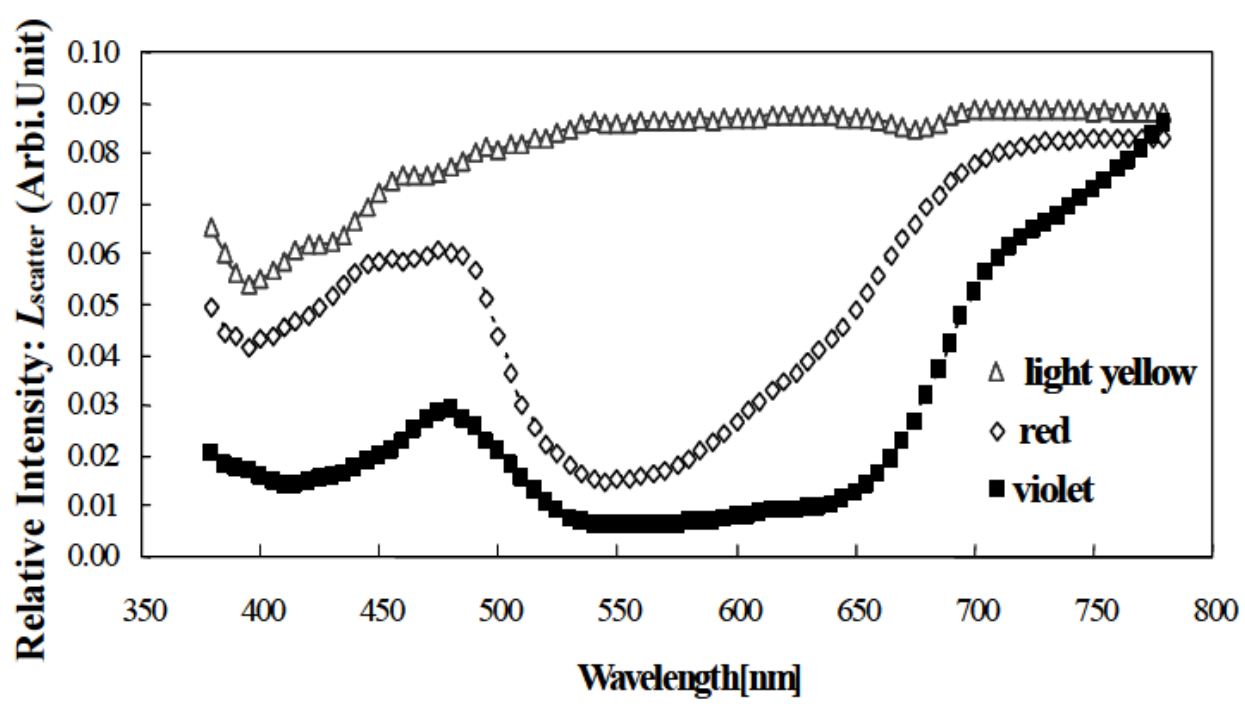

(a)

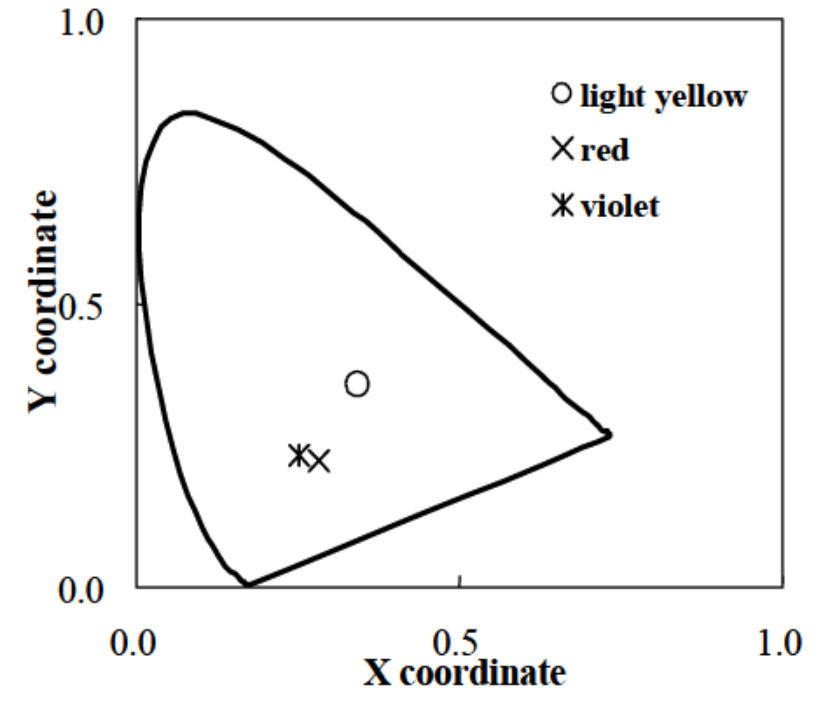

(b)

Fig. 2. (a) Measured spectra of normalized scattered lights from light yellow, red, and violet .petals and (b) Chromaticity coordinates following each measured spectrum. 
We must confirm whether these obtained spectra correspond to each color of petals. Using these spectra, we calculate the chromaticity coordinate [4]. The calculated coordinates are shown in Fig. 2 (b), where each point matches the original color of petals.

It is assumed that the scattering light spectrum is formulated as follows;

$$
\mathrm{L}_{\text {scatter }}(\lambda)=\mathrm{L}_{\text {irradiate }}(\lambda)-\mathrm{L}_{\text {reflect }}(\lambda)-\mathrm{L}_{\text {absorb }}(\lambda)-\mathrm{L}_{\text {transmit }}(\lambda),
$$

where $\mathrm{L}_{\text {scatter }}$ is for the scattered light intensity, $\mathrm{L}_{\text {irradiate }}$ is for the irradiated light intensity, $\mathrm{L}_{\text {reflect }}$ is for the direct-reflection light intensity, $\mathrm{L}_{\mathrm{absorb}}$ is for the absorbed light intensity, and $\mathrm{L}_{\text {transmit }}$ is for the transmitted light intensity. We neglect the second emission by absorption here, for it may be little effect on this analysis. As the measured spectrum of the scattered light is normalized by the irradiated light, therefore Eq. (1) is rewritten as follows;

$$
L_{\text {scatter }}(\lambda)=1-L_{\text {reflect }}(\lambda)-L_{\text {absorb }}(\lambda)-L_{\text {transmit }}(\lambda),
$$

where $L$ means the normalized light intensity.

As we have mentioned, obtained spectra include little the direct-reflection light and $L_{\text {reflect }}$ can be neglected. As the samples are thick, $L_{\text {transmit }}$ can also be neglected. The intensity of the scattered light is proportional to the intensity of the irradiated light. Therefore, the subtraction of the scattering spectrum of the red petal from the light yellow one is considered to show the change in the absorption spectrum.

Figure 3 (a) shows the spectrum according to the subtraction between the spectra of light yellow and red petals. This spectrum corresponds to the absorption spectrum caused by the red petal, with the peak wavelength of $545 \mathrm{~nm}$.

It is shown in Fig. 3(b), the spectrum according to the subtraction between the spectra of red and violet petals. It is found that there are two absorption peaks at $450 \mathrm{~nm}$ and $670 \mathrm{~nm}$ wavelengths, which are caused by the violet petals. From the scattered spectra for the violet petal in Fig. 2 (a), the dip wavelength at $450 \mathrm{~nm}$ is difficult to be observed. It is found just after this subtraction. These three absorption peaks contribute to the color of flowers' petals.

Figure $3(\mathrm{c})$ and (d) are the measured absorbance spectra for the light yellow petal and the red petal, respectively. In this measurement, we have used the mashed petals dissolving in solvent: the dilute hydrochloric acid with methanol and water. As shown in Fig. 3 (c), there is little absorption change in the visible region. The fact that the absorbance for the light yellow petal is larger than that for red petal is consistent with the results shown in Fig. 2 (a). Fig. 3(d) shows the absorption with peak wavelength around $550 \mathrm{~nm}$, which is similar to the result shown in Fig. 3 (a). In Ref. [8, 9], red petals were analyzed by using HPLC: High performance liquid chromatography by observing the absorption for $530 \mathrm{~nm}$ light incident. From these results, it is found that the red petals have the absorption around $550 \mathrm{~nm}$, and it is confirmed that our proposed method can derive the absorption spectrum. 

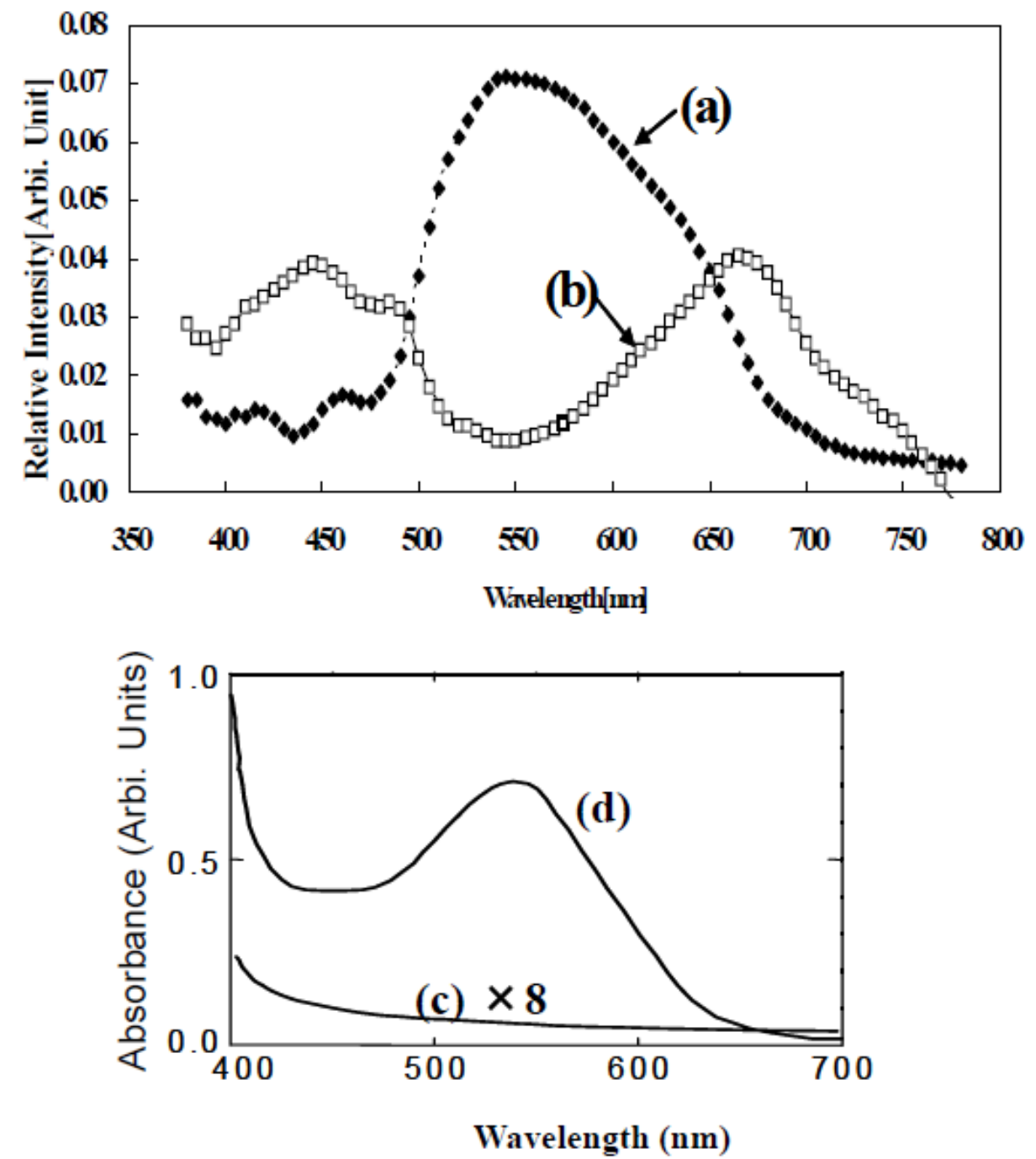

Fig. 3. Estimated spectra of absorption after the subtraction of scattered light spectrum (a) of the red petal from that of the light yellow one, (b) of the violet petal from that of the red one, and the absorbance spectrum (c) of the light yellow, and (d) of the red petal.

We have tried to calculate the trace of the movements on the color coordinates by changing the magnitude of three absorption spectra. We have confirmed that the movement from the light yellow point to red point on the chromaticity coordinates as shown in Fig. 2 (b) according to the increase in the absorption with the peak wavelength of $545 \mathrm{~nm}$ and from the red point to violet point according to the increase in the absorption with the peak wavelengths of $450 \mathrm{~nm}$ and $670 \mathrm{~nm}$. If the absorption peak at $450 \mathrm{~nm}$ is neglected, the movement from the pink point can not reach the violet point. When this absorption increases further, the calculated movement reaches the blue color coordinates.

The color of petals is determined by the acylated form of pigments in cells of petals. The pigment included in the petunia hybrida is reported antocyanins $[5,6,7]$. The antocyanin is reported to be biosynthesized in cells 
of petals from two materials of coumaronyl CoA: coenzyme A and malonyl $\mathrm{CoA}$ and the type of the bio-synthesized antocyanin is antocyanidin $3 \times$ glucoside. There are three kinds of antocyanidin according to the number of $\mathrm{OH}$ radials, where antocyanidin with two $\mathrm{OH}$ radicals is related to the red color and that with three $\mathrm{OH}$ radicals is related to the blue color. That of one $\mathrm{OH}$ radical causes brown or brick color but it is very difficult to biosynthesize in petunia hybrida [10]. Moreover it is reported that the background $\mathrm{pH}$ values are different for each color; that is acid for red, neutral for violet, and alkali for blue [5]. In the Ref. [6], the antocyanins acylated with caffeic acid are found in red and pink petals of petunia hybrida. In the light yellow petals, the antocyanin is just under biosynthesizing. It is the flavanone which is made during the bio-synthesizing. It hardly absorbs the visible light as shown in Fig. 3 (c). It makes the color of petal light yellow or sometimes white as shown in the Fig. 1 (c).

The change in the absorption spectra depends on the different acylated form of antocyanin. From our results, the intensity of scattered light intensity decreases according to light yellow, red, and violet. The subtraction between two spectra suggests the appearance of the additional absorption peaks. About the appearance of the color of petals, one hypothesis is proposed according to our results as follows. It is assumed that the antocyanidin with three $\mathrm{OH}$ radicals is biosynthesized simultaneously that with two $\mathrm{OH}$ radicals. First the antocyanidin with two $\mathrm{OH}$ radicals exists alot and the color of petals becomes red. When both quantities balance, the color of petals becomes violet, and when that with three $\mathrm{OH}$ radicals is superior to that with two $\mathrm{OH}$ radicals, the color of petal changes to be blue. Our results will help the consideration of the colorization of petals of the petunia hybrida.

\section{Summary}

We have investigated the new method to evaluate the absorption spectrum by using the scattered light spectrum. In this method the key issue of measurement set-up is to prevent the direct-reflection light which includes the irradiated light source spectrum. By using this method, we have evaluated the petals of petunia hybrida. We have derived the absorption spectrum from the scattered light spectra and have found three absorption peaks of these petals.

Our proposed method is effective to evaluate the absorption without changing the shape of samples and to evaluate the mechanism of the change in color of flowers' petals of the identical species.

\section{Acknowledgement}

We have expressed the appreciation to Fujitsu Laboratories Ltd. for their kind help for measurements. 